\title{
Innovación y Producción en la Industria Manufacturera: Estudio Comparativo de Cadenas Globales
}

\author{
Innovation and Production in Manufacturing Industry: a Comparative Study \\ about Global Chains
}

\author{
Sebastián Sztulwark', Santiago Juncal ${ }^{2}$
}

\begin{abstract}
Desde hace cuatro décadas se verifica la emergencia de una nueva lógica de acumulación en el seno de la producción capitalista. La hipótesis del trabajo es que en la industria manufacturera esta nueva tendencia se expresa en la creciente segmentación de las etapas de innovación y fabricación de productos, acompañada de una integración de las variantes informacional y estético-expresiva en el plano de la innovación. A partir de un abordaje que combina diferentes categorías teóricas (cadenas de valor global, sistema histórico de acumulación, desacople entre capacidades de innovación y producción) y de una metodología basada en el análisis de fuentes secundarias, se estudiaron las cadenas farmacéutica, de indumentaria y de productos electrónicos de consumo. Los resultados alcanzados permitieron confirmar la hipótesis planteada y la detección de particularidades en materia de naturaleza de la innovación, constitución de barreras a la entrada y dispersión geográfica para cada cadena.
\end{abstract}

Keywords: cadenas productivas globales; innovación; industria manufacturera.

A new logic in the capitalist accumulation process has emerged in the last four decades. The hypothesis of the article is that this new logic expresses itself in the manufacturing industry through the increasing segmentation of product innovation and product manufacturing activities, and the integration of two alternative types of innovation activities: informational and aesthetic-expressive. Starting from an approach that combines different theoretical categories (global value chain, historical system of accumulation, disconnection between innovation and production capabilities) and a methodology based on the analysis of secondary sources of information, three productive chains have been studied: pharmaceutical, apparel and consumer electronics. The outcome of the research confirms the formulated hypothesis and allows us to detect specificities in the content of innovation, the constitution of entry barriers and the geographical dispersion in each chain.

Keywords: global commodity chain; innovation; manufacturing industry.

\footnotetext{
1,2 Instituto de Industria de la Universidad Nacional de General Sarmiento. J.M. Gutiérrez II50, Los Polvorines, Provincia de Buenos Aires, Argentina; CP 16I3. e-mail: 'ssztulwa@ungs.edu.ar, ${ }^{2}$ sjuncal@gmail.com
}

ISSN: 07 I8-2724. (http://www.jotmi.org) 


\section{Introducción}

Desde hace aproximadamente cuatro décadas, es posible verificar la emergencia de una nueva lógica de acumulación en el seno del modo de producción capitalista. Esta transformación tiene como eje un desplazamiento de la creación de valor desde una modalidad de reproducción de bienes de baja diferenciación hacia un régimen progresivamente sesgado hacia la innovación de producto y en el que, por lo tanto, emerge un nuevo rol del conocimiento y la innovación en los procesos de valorización. Esta ruptura histórica ha sido catalogada de diferentes maneras por diversas corrientes teóricas ("capitalismo pos-industrial", "capitalismo cognitivo", "capitalismo informacional", etc.) y el debate sobre su naturaleza y alcance permanece aún vigente.

En este contexto, el carácter jerárquico y asimétrico de la economía mundial no desaparece, pero debe ser repensado. Dos elementos fundamentales para entender estas transformaciones son, por un lado, los procesos de segmentación y dispersión global de la producción y, de forma simultánea, la centralización del comando en torno a la figura de las firmas líderes de las cadenas de valor global. Tomados estos elementos en consideración, es posible aseverar que las asimetrías entre diferentes territorios pasan a residir ya no sólo entre los tipos de bienes o servicios que cada uno produce, sino más bien en la posición de la cadena en que se ubiquen las actividades económicas que se desenvuelven en cada una de esas unidades territoriales (Gereffi, 200I; Kaplinsky, 2000; Dicken, 2003).

En el marco de esta problemática general de investigación, en este trabajo nos interesa pensar la relación entre la emergencia de nuevas modalidades de valorización capitalista y la reconfiguración de la industria manufacturera a nivel mundial, con el objetivo de avanzar en la comprensión de los nuevos patrones de segmentación productiva global que se despliegan al interior de ese sector.A modo de hipótesis, los elementos fundamentales a considerar son: i) que la base de esta segmentación global industrial se corresponde con el despliegue de dos modalidades de valorización complementarias pero cualitativamente diferenciadas: por un lado, las actividades de innovación, basadas en la creación de nuevo conocimiento (novedad de alcance mundial) que tienen un papel dominante; por otro, las actividades de producción, orientadas a la reproducción de conocimiento existente, que juegan un papel subordinado; ii) en la modalidad dominante, la innovación no se agota en las mejoras de tipo informacional (conocimiento científico y tecnológico, abstracto y codificado), sino que juegan un papel de creciente relevancia los elementos "estético-expresivos", esto es, aquellos orientados a movilizar los elementos emocionales que vinculan al consumidor con el producto; iii) sobre esta base, se despliega un nuevo patrón geográfico de localización industrial en el cual las actividades de innovación se concentran en los territorios en los que existen sistemas de innovación desarrollados y las actividades de producción se difunden a zonas en las que prevalecen menores costos unitarios de producción.

Para avanzar en estas hipótesis, se analizan tres cadenas industriales específicas: farmacéutica, electrónica de consumo e indumentaria y prendas de vestir. Estas cadenas comparten al menos tres denominadores comunes que posibilitan la comparación: elaboran bienes de consumo final que admiten cierto grado de diferenciación de producto; implican actividades susceptibles de segmentación productiva (es decir, no involucran procesos de flujo continuo); y experimentan algún nivel de dispersión global. La metodología empleada es de tipo comparativo, a partir de la identificación de las dimensiones críticas principales para el análisis de las cadenas. Es trabajo se apoya parcialmente en fuentes primarias de información (datos provistos por las empresas, sobre todo en sus páginas web) y mayormente en fuentes secundarias, como trabajos académicos sectoriales y material de prensa especializado.

El trabajo se estructura de la siguiente manera. En primer lugar, se presenta el marco teórico-metodológico. Luego se desarrolla el análisis de cada una de las cadenas siguiendo los ejes conceptuales definidos en el apartado previo: la caracterización de los diferentes procesos de innovación, de las barreras a la entrada principales de cada eslabón y del patrón geográfico que se despliega en cada caso a nivel global. El trabajo cierra con una breve reflexión final.

\section{Marco teórico}

El marco conceptual del trabajo se sostiene en una serie de dimensiones. En primer lugar, para abordar el cambio histórico consideramos necesario tomar el concepto de sistema histórico de acumulación, desarrollado por Dieuaide, Paulré y Vercellone (2007), el cual alude "a la asociación entre un modo de producción y una lógica de acumulación que orientan en un largo período las tendencias de la valorización del capital, la división del trabajo y la reproducción de las relaciones sociales más fundamentales" (p. 74). Así, el "nuevo capitalismo" refiere al sistema histórico de acumulación específico que surge a nivel mundial en el último tercio del siglo $X X$, a partir de la crisis del capitalismo industrial, que fue el sistema de acumulación surgido con la Revolución Industrial y que dio lugar a la sustitución de la producción artesanal por un sistema orgánico de máquinas-herramienta operadas por trabajadores descalificados a partir del impulso de fuentes de energía inanimadas (Dabat, 2009). 
La emergencia del "nuevo capitalismo" implica un cambio en el papel del proceso de innovación en el seno del sistema. Esto se expresa, en primer término, en el hecho de que la innovación de producto pasa a tener una centralidad que en el capitalismo industrial recaía en las innovaciones de proceso (Sztulwark, Míguez y Juncal, 20l I). Asimismo, la innovación de producto se materializa en una creciente intensidad del diseño que -a su vez- está relacionada con la potenciación del componente "reflexivo" del consumo en este período histórico. La idea de "intensidad de diseño" es expuesta por Lash y Urry (1998) cuando sostienen que "la producción no sólo está más penetrada de conocimiento, sino que se ha vuelto más cultural". Así, la innovación adquiere un carácter no sólo informacional, sino también estético-expresivo; para realizar una ganancia extraordinaria por innovación ("renta de innovación"), los bienes y servicios deben estar cada vez más provistos de un "componente estético", sobre el que descansa en última instancia su "valor de signo"'.

Estos cambios se corresponden, a su vez, con la existencia de un desacople entre producción e innovación en el nuevo capitalismo ${ }^{2}$. Las capacidades de producción son aquellas que se orientan fundamentalmente hacia el uso y la adaptación del conocimiento existente. Según Bell y Abu (1999), estas capacidades se vinculan con la replicación y circulación de conocimiento que ya está establecido dentro del sistema de producción, con la potencialidad de expandir la capacidad productiva usando métodos de producción dados. Por otro lado, el desarrollo de capacidades de innovación tiene como núcleo la adquisición, creación, procesamiento y acumulación de nuevo conocimiento. Esta diferenciación entre tipos de capacidades nos permite identificar las diferentes alturas de las barreras a la entrada en cada caso. Concretamente, es posible sostener no sólo que las barreras a la entrada en las actividades de innovación (umbral de inversión en activos intangibles) son tendencialmente superiores a las verificadas en las actividades de producción (costo unitario de producción), sino que además son cualitativamente diferentes $y$, por tanto, que la acumulación de capacidades productivas no necesariamente conduce a un dominio de las de innovación (Altenburg et al, 2008).

El panorama se completa tomando en consideración el correlato geográfico de estos procesos. En otras palabras, la posibilidad de segmentar físicamente los procesos de innovación y producción se traduce en la actualidad en una dispersión a escala global de dicha escisión. La segmentación productiva global no constituye en sí misma un fenómeno nuevo; lo novedoso es que a partir de los avances tecnológicos, organizativos e institucionales recientes, esta fragmentación de la cadena de valor adquiere una gran complejidad y un alcance internacional, configurando una de las tendencias fundamentales de la globalización ${ }^{3}$. En otras palabras, la dinámica de la competencia en las últimas décadas se transformó tanto en extensión como en complejidad.
La dispersión de las actividades que componen las cadenas queda sujeta, en última instancia, a diferentes formas de coordinación, regulación y gobierno. Dicken (2003) define a las cadenas productivas como secuencias de funciones transaccionalmente conectadas en las que cada etapa agrega valor al proceso de producción, y afirma que se encuentra en marcha un proceso de creciente especialización vertical (inverso al de la integración vertical) y fragmentación del proceso productivo. Las empresas líderes son las que logran ejercer el comando (governance), la capacidad de dominar los aspectos principales del proceso productivo (parámetros o estándares de producto, escala, tiempo, etc.) sobre sus empresas subordinadas y de controlar la distribución de la renta al interior de la cadena. Gereffi (200I) presenta la distinción entre dos tipos de cadena global de producción, según el comando sea realizado por el productor o el comprador. El primer tipo de cadena remite a industrias con formas organizativas tradicionales, que formaron parte del núcleo del patrón productivo fordista, como la industria automotriz, de aviones o de maquinaria. En cambio, en las cadenas comandadas por el comprador la clave del comando no deriva del control tecnológico, sino de una serie de capacidades complementarias de investigación, diseños, ventas, marketing o servicios financieros.

Con todo, la caracterización de los diferentes tipos de comando se vincula profundamente con el tipo de despliegue territorial que adquieren las cadenas. En última instancia, el mismo concepto de cadena productiva debe ser asimilado a una determinada configuración geográfica de relaciones económicas entre territorios con distintas capacidades productivas e innovativas. Desde ya, la existencia de distintos niveles de acumulación de estas capacidades obedece a cuestiones históricas de diversa índole, que incluyen naturalmente la presencia de mecanismos autorreforzados. Lo relevante para nuestro análisis radica en que esas asimetrías reflejan sistemas de conocimiento disímiles, cuyo relacionamiento es la expresión territorial del desacople al que refieren algunos de los autores arriba citados.

'Nuevamente en palabras de Lash y Urry:“En la producción, el componente de diseño forma una proporción cada vez más grande del valor de los bienes. El proceso específico de la fuerza de trabajo pierde importancia en el aporte al valor agregado, y la adquiere, en cambio, el "valor de diseño"”. ${ }^{2} E$ sta distinción no está exenta de problemas, ya que innovación y producción están entrelazadas: producir implica cierto grado de innovación y la innovación está articulada con la producción. La distinción es analítica y alude, por un lado, a las capacidades necesarias para producir (o reproducir) bienes existentes y, por otro, a la capacidad para diferenciar o crear nuevos productos. En un caso, el eje es la productividad, la brecha de costos en relación al productor líder mundial; en el otro, es la capacidad de imponer nuevos diseños dominantes (en el sentido de Abernathy y Utterback, 1978). ${ }^{3} \mathrm{El}$ proceso de fragmentación global no es universal. Excluye a las industrias de proceso continuo y a muchos servicios. Sin embargo, su importancia deriva de que las industrias que constituyen el núcleo del nuevo patrón productivo, que es el que dinamiza al conjunto de la economía, están sujetas a esta fragmentación (Rivera, 2005). 
Sobre esta línea argumental podemos afirmar que, en el actual contexto histórico, existen nuevos fundamentos cognitivos de la valorización capitalista que pueden resultar útiles para comprender los procesos de segmentación en la industria manufacturera mundial. Los elementos fundamentales a considerar son:i) la preeminencia económica de las actividades de creación de nuevo conocimiento (innovación) por sobre aquellas orientadas a la reproducción del conocimiento existente (producción); ii) el protagonismo creciente de la dimensión estético-expresiva en las actividades de innovación, que complementan (y no sustituyen) a aquellas que aluden a la dimensión informacional (conocimiento codificado y abstracto); iii) la conformación de un nuevo patrón geográfico mundial, en el que las actividades de innovación tienden a concentrase en los países que cuentan con sistemas de innovación más desarrollados mientras que las actividades de producción tienden a localizarse en territorios cuya ventaja está íntimamente ligada a la obtención de mínimos costos unitarios de producción.

En las páginas que siguen intentaremos verificar -a través de tres casos específicos diferentes- en qué medida estas tendencias operan efectivamente en la dinámica de la industria manufacturera a nivel mundial $y$, por lo tanto, identificar la existencia de elementos de contra-tendencia que puedan enriquecer las bases de nuestra comprensión de los fenómenos de segmentación económica global.

\section{La cadena de la indumentaria y prendas de vestir}

Según Gereffi y Memedovic (2003), la configuración de la cadena de producción de prendas de vestir incluye la presencia de cinco eslabones principales: materias primas (algodón y lana en fibras naturales, y petróleo y gas en fibras sintéticas), componentes textiles (fibras sintéticas y naturales y su hilado y tejido), fabricantes de prendas (diseño, corte, costura, abotonado y planchado), exportadores y comercializadores (operada por empresas con marca, distribuidoras y comercializadoras) y comercialización minorista (grandes almacenes, tiendas de especialidades, cadenas de comercio masivo, outlets y otros). Si bien existen perspectivas alternativas a esta caracterización (Padilla et al, 2008; Stanley, 20 I I), los matices entre los diferentes autores no implican diferencias sustantivas en el análisis.

En términos de innovación, tanto Kaplinsky (2005) como Padilla et al (2008) coinciden en indicar que el avance tecnológico mas trascendente de la cadena se ha producido en la actividad de comercialización, mediante la implementación de sistemas de códigos de barras y de TICs asociados, lo que permitió identificar la magnitud de los stocks de distintos productos y reorientar la producción en base a ello, generando bajas de costos y proveyendo variedad de bienes a mayor velocidad. La literatura destaca asimismo el escaso nivel de innovaciones tecnológicas que tuvo lugar en el eslabón de la fabricación desde su misma aparición en el siglo $X I X^{4}$ y las numerosas iniciativas implementadas en los últimos años en la producción de insumos textiles, en nuevos procesos de hilado y tejido y en tipos de tela para diferentes sectores (Padilla et al, 2008).

Además de destacar las sofisticadas y costosas innovaciones enTICs, Gereffi y Memedovic (2003) indican que las empresas líderes cuentan también con "abundantes presupuestos para publicidad y campañas promocionales necesarios para crear y sostener marcas globales"5. Estas dos dimensiones de la innovación resultan cruciales fundamentalmente en las sub-cadenas lideradas por distribuidoras, tiendas $y$ comercializadoras masivas. En tanto, en los casos de subcadenas donde las firmas líderes combinan la presencia de marcas de prestigio internacional y ventas de productos de alta gama, la innovación vía diseño de calidad constituye el otro activo estratégico insoslayable e inseparable de los desembolsos publicitarios ${ }^{6}$.

Como trasfondo de estos elementos explicativos, Dicken (2003) destaca dos procesos claves en el seno de la cadena. Uno de ellos es la "revolución minorista" que tuvo lugar en los países desarrollados a partir de la década de 1960, la cual se complejizó hacia una segmentación de mercados (por edades y niveles de ingreso) desde la década de 1980 en adelante. Los supermercados y las cadenas de descuento son, naturalmente, las empresas más asociadas a estos movimientos. El otro elemento es la aceleración en los cambios en la moda, que son inducidos por las compañías con marca y/o diseños reconocidos en el mercado y tienden a ser fundamentales. Stanley (20I I) ratifica esto, al destacar la necesidad de "adaptarse a los cambios en los gustos de los clientes y tener el mejor diseño", toda vez que "las temporadas se acortan en duración, sumándose nuevas colecciones todo el año, alta rotación y diversidad"7.

${ }^{4}$ Dicken (2003) indica que la manufactura de prendas continúa basándose en operaciones manuales interconectadas, mientras que Padilla et al (2008) coincide al sostener que "en el eslabón de la manufactura o confección no ha habido grandes cambios" tecnológicos.

${ }^{5}$ Según Interbranding, de las 100 marcas de mayor cotización a nivel mundial en 20II, diez de ellas operan en el sector de indumentaria: Louis Vuitton, H\&M, Nike, Gucci, Zara, Adidas, Hermes, Gap, Armani y Burberry. Sus cotizaciones van desde los U\$S 23.000 millones (Louis Vuitton) hasta los U\$S 3.700 millones (Burberry).

${ }^{6}$ Kaplinsky (2005) resume esto al sostener que "las rentas derivadas del diseño están cada vez más vinculadas a las rentas derivadas del marketing y a las inversiones en el sostenimiento de marcas".

${ }^{7}$ Gibbon (2008) sostiene que en ciertos segmentos las temporadas de moda han pasado de 4 a 6 en un año, lo que se traduce en la reducción del tiempo de elaboración de un rango de 5-6 meses a uno de l-3 meses promedio. 
Al analizar las barreras a la entrada en la cadena de prendas de vestir, Gereffi y Memedovic (2003) indican que éstas son bajas en los establecimientos confeccionistas (eslabón trabajo-intensivo y con pocos requerimientos de capital físico) y algo más elevadas en los de insumos textiles y fibras, que es altamente automatizada y opera con economías de escala. En sintonía con lo expresado más arriba, las barreras se elevan aun mas en los grandes comercializadores globales; éstos exhiben sus principales activos competitivos en la posesión de marcas y diseño de alta calidad (sobre todo en las compañías orientadas por la moda y las empresas "con marca") y en sus capacidades organizacionales para administrar la cadena (fundamentalmente, supermercados y cadenas de descuento).

Estas capacidades se generan, a su vez, a partir de la ya mencionada incorporación de tecnologías de información. Gereffi y Memedovic (2003) denominan "venta minorista racionalizada" ("lean retailing") al modelo organizacional correspondiente, al cual se considera un factor importante en el desplazamiento de los industriales tradicionales (branded manufacturers) como líderes de la cadena. En su análisis del mercado estadounidense, Gereffi (1999) diferencia a éstos de otros tipos de empresa líder, como los minoristas (retailers) y las empresas con marca (branded marketers $)^{8}$. Esta categorización es útil para indicar que, mientras los tres grupos de empresas cumplen el mismo rol estructural de ser grandes compradores líderes de la cadena, los branded manufacturers son los únicos que requieren que sus proveedores se dediquen al ensamblaje de partes cortadas para lograr el producto final; los minoristas y los marketers se aprovisionan, en cambio, de firmas con ciertas capacidades productivas pero también logísticas que le permitan subcontratar insumos a otras firmas locales.

El matiz marcado por Gereffi (1999) se complejiza aun más en Gibbon (2008), quien sostiene que la conducción del aprovisionamiento es una barrera a la entrada en el eslabón de la comercialización que tiene también implicancias directas a lo largo de la cadena. Así, los proveedores "de primer nivel" deben generar innovaciones en la reorganización de la cadena para establecer grupos de proveedores con "altos niveles de capacidad productiva, bajos precios, capacidades complementarias para manejar sus tiempos de entrega y acceso a preferencias comerciales". El objetivo es mejorar su posición relativa con relación a las firmas líderes, poniendo de manifiesto que las capacidades organizacionales no son exclusivamente detentadas por aquellas. Asimismo, los productores de "segundo nivel" también deben cumplir una serie de capacidades funcionales y requerimientos para mantenerse competitivos ${ }^{9}$, lo que revela en última instancia la incidencia de la sofisticación de la demanda final.
Este fenómeno da cuenta de una tendencia al crecimiento en las barreras a la entrada en el eslabón confeccionista, lo cual no debe ocultar el verdadero trasfondo en el que se verifica: la permanencia de bajos costos salariales como variable clave para comprender la globalización del sector $y$, en definitiva, de amplísimas diferencias de altura entre las barreras de las etapas de comercialización y fabricación. En definitiva, fenómenos como la creciente importancia de la producción textil china en los últimos años del siglo pasado y la más reciente aparición de países competidores como Bangladesh (en el marco de elevados niveles de explotación laboral en empresas proveedoras de las grandes marcas globales) no encuentran explicación sino en las bajas remuneraciones vigentes en dichos territorios.

Los escasos avances tecnológicos y los bajos costos de iniciación en la confección han contribuido a generar un alto grado de globalización de dicho eslabón. Gereffi (1999) y Padilla et al. (2008) coinciden en reconocer tres migraciones sucesivas en la producción mundial confeccionista tras el fin de la Segunda Guerra Mundial. La primera tuvo lugar en las décadas de 1950 y 1960 desde EEUU y Europa Occidental hacia Japón, la segunda se produjo a lo largo de las dos décadas siguientes desde Japón hacia Corea, Taiwán y Hong Kong, y la tercera se efectivizó durante la década de 1990 desde estos territorios hacia diferentes regiones de países en desarrollo (Europa del Este y América Central, fundamentalmente) cercanos a los principales centros de consumo mundiales. Antes de este último viraje, el crecimiento de la producción había ubicado a China como primer proveedor de indumentaria a nivel mundial.

\footnotetext{
${ }^{8}$ Kaplinsky (2005) define a los branded manufacturers como productores que históricamente han fabricado sus propios productos, pero que progresivamente pasaron a aprovisionarse de los mismos en cadenas independientes pero fuertemente coordinadas, generalmente triangulando la función de coordinar y comprar en un país, con la elaboración emplazada en un tercero.

'Gibbon (2008) enumera una serie de ellos, entre los que se hallan la presencia de capacidad productiva mínima, la flexibilidad para aumentar el volumen producido y reducir los tiempos de entrega y la separación sistemática de los insumos de distintos clientes.
} 
En sintonía con lo ya expuesto, resulta evidente que en estas relocalizaciones han confluido también razones de oferta (bajos costos salariales) y de demanda (necesidad de responder con velocidad y variedad a preferencias cada vez más sofisticadas). Los costos diferenciales salariales tuvieron mayor incidencia en las dos migraciones iniciales, mientras que la necesidad de responder eficientemente a las nuevas tendencias del consumo explica en mayor medida el último proceso migratorio. Asimismo, factores institucionales también han influido en este fenómeno; la creación de la Unión Europea y el NAFTA condujo al establecimiento de aranceles preferenciales al interior de ambos bloques, lo que generó una consolidación de cadenas de aprovisionamiento al interior de las regiones (Gereffi, 1999). Como sostiene Stanley (20II), esto generó una dinámica coexistencia de cadenas regionales e internacionales ${ }^{10}$.

Por su parte, la acumulación de capital y poder de los grandes compradores globales revela matices según la ubicación geográfica. Dicken (2003) apreciaba que en las compañías orientadas por la moda coexistía la presencia de empresas europeas con marcas reconocidas (Armani, Boss, Gucci) con algunas estadounidenses (Ralph Lauren, Donna Karan). En tanto, la mayoría de las tiendas con marca (Bloomingadale's, Macy's, The Gap, Liz Clairborne y Calvin Klein), supermercados (Sears, Montgomery Ward y J.C. Penney) y cadenas de descuento (Wal-Mart, Kmart y Target) era estadounidense. Indicio de la continuidad de su liderazgo, muchas de estas firmas son también mencionadas en trabajos más recientes, como el de Padilla et al (2008).

La presencia europea resulta significativa en los casos de grandes marcas deportivas alemanas (Adidas y Puma) y de firmas italianas y francesas en compañías de moda de alta gama (Lacoste, Prada, Hermès, Versace, etc.), pero también entre las grandes cadenas de comercio minorista a nivel mundial. Si se consideran las diez firmas minoristas con mayor facturación en 2010 y con algún nivel significativo de operación en la venta de prendas de vestir, se observa que dos son estadounidenses, siete europeas (cuatro alemanas, dos francesas y una británica) y una japonesa". Esto no obsta el liderazgo indiscutido de la estadounidense Wal-Mart, cuyas ventas (U\$S 416.000 millones) más que triplicaron a la de su inmediato seguidor (Carrefour), por lo que es considerada con el suficiente poder de mercado como para "establecer precios e influir sobre la distribución geográfica de las operaciones de confección" a nivel global (Padilla et al, 2008).

\section{La cadena de la industria electrónica de consumo}

Dicken (2003) señala que la elaboración de productos electrónicos de consumo consiste en tres etapas: diseño, manufactura de componentes y ensamble. De las tres, el autor destaca a la primera de ellas como la más intensiva en investigación. Este planteo debe ser complejizado con perspectivas alternativas más recientes. Sturgeon (2002), por caso, afirma que en la industria electrónica se impone progresivamente un esquema de "redes de producción modular", en el que las firmas líderes son poseedoras de marcas mundialmente reconocidas, cuya competencia central consiste en concentrarse en crear, penetrar y defender mercados para productos finales, llevando a cabo a tal efecto actividades de estrategia, definición, desarrollo, diseño y marketing de producto.

Por su parte, Dedrick y Kraemer (2006) analizan el caso particular de la producción de PCs portátiles, identificando tres fases productivas a las que denominan diseño, desarrollo y producción, y desagregando cada una de ellas en distintas sub-fases. Las empresas multinacionales con marca reconocida son las que se concentran en el diseño de productos, subcontratando la fase productiva y, en buena medida, los procesos de desarrollo de los productos. Esta caracterización nos conduce a la necesidad de ligar el papel de la innovación a los distintos modelos de negocio, que en la práctica se verifican bajo estrategias empresariales diversificadas: firmas taiwanesas que fabrican para terceros pero también bajo propia marca (Sturgeon, 2002) y el diseño/ desarrollo conjunto llevado a cabo entre empresas con diseño propio y firmas con marca líder (Dedrick y Kraemer, 2006) son ejemplos de estas combinaciones ${ }^{12}$.

\footnotetext{
${ }^{10} \mathrm{El}$ autor explica esto al sostener: "El ahorro salarial puede resultar caro. La distancia que existe entre China y Europa juega en la misma dirección. Esto introduce un nuevo mix: costos salariales - distancia" (Stanley, 20I I). "Datos extraídos del siguiente sitio: http://www.stores.org/20II/Top250-List

${ }^{12}$ Otro caso es el de Samsung, que provee a Apple de componentes que explican conjuntamente el $26 \%$ de los costos de su teléfono móvil; así, "Apple es uno de los clientes más grandes de Samsung, y Samsung es uno de los proveedores más grandes de Apple" (The Economist, 20II). Pero Samsung también comercializa tablets y teléfonos inteligentes, por lo que termina siendo proveedor de uno de sus principales competidores.
} 
El trasfondo de estas relaciones altamente complejas se aprecia en el rejuvenecimiento tecnológico de los productos y la integración de sus funciones, dos tendencias que resultan ser complementarias y a la vez contradictorias. Ambos fenómenos se ha verificado en múltiples bienes, como televisores (Dicken, 2003), cámaras fotográficas (auge de las unidades digitales compactas sin espejo), teléfonos celulares y computadoras (ambos adquiriendo diversas aplicaciones, como mensajería, juegos, notificaciones, servicios de georreferenciación, etc. ${ }^{13}$ ). No obstante, la integración de funciones evidencia límites precisos, dada la imperfecta sustitución que continúa existiendo entre los diferentes bienes, como se verifica entre las tablets y las laptops.

La innovación en diseños y comercialización de producto se hallan, así, en el núcleo de las estrategias de las empresas líderes. Recientes experiencias fallidas en Sony (creador de productos sólidos tecnológicamente, pero poco funcionales), Nokia (desconexión entre áreas de I+D y comercialización) y H-P (debilidad en diseño de productos) resultan ilustrativas de este fenómeno (The Wall Street Journals, 20I2b, 20I2c y 20I2e). La evidencia indica que la generación de diseños estéticos y funcionales debe apoyarse en una estrategia de comercialización y posicionamiento de marcas acorde a dichas innovaciones. En suma, la innovación de las empresas con marca contempla desde ya la dimensión informacional (solidez tecnológica de los productos), pero con un componente estético y simbólico que pesa crecientemente, como el caso de Apple y el consumo reflexivo de sus productos ${ }^{14}$, destacado por autores como Dedrick, Kraemer y Linden (2009) ${ }^{15}$.

Los planteos de Dicken (2003), Dedrick y Kraemer (2006) y Sturgeon (2002) detentan utilidades complementarias. El aporte del primero sirve para comprender que los componentes manufacturados pueden ser de carácter pasivo (resistencias, cables, conectores, etc.) o activo (semiconductores, microprocesadores, etc.), más complejos y generalmente fabricados por proveedores especializados; Dedrick y Kraemer explican las formas que puede adquirir la relación entre empresas con marca y diseño propio en los procesos productivos, y Sturgeon aporta una valiosa caracterización de los proveedores de las empresas con marca, al indicar que su competitividad se basa en la posesión de una cartera diversificada de clientes, para lo que requieren desarrollar servicios y capacidades genéricas. A esto se suman otros factores, como los bajos salarios, la férrea disciplina laboral en las fábricas y la capacidad de respuesta a demandas puntuales de las empresas con marca, los cuales explican la acentuada radicación en China ${ }^{16}$.

El hecho de que la cadena de electrónica de consumo es intensiva en gastos en I+D se aprecia en diversas estadísticas generales sobre el sector. Datos de la National Science
Foundation de los EE.UU. indican que la investigación y desarrollo como porcentaje de las ventas fue más elevada en el segmento "Computadoras y productos electrónicos" que en cualquier otro de los involucrados entre las actividades industriales en el período 1999-2008.

La información sobre gastos en I+D de empresas individuales también son de elevada magnitud. En 201 I, las inversiones en I+D de Sony, Panasonic y Samsung alcanzaron los 5.500, 6.600 y 8.700 millones de dólares, respectivamente ${ }^{17}$. Por su parte, la estadounidense Apple viene incrementando sostenidamente sus gastos en I+D desde 2006, aunque el monto alcanzado en 2010 no superó los U\$S 2.000 millones. Esta magnitud relativamente baja para la industria electrónica se replica en términos relativos; Apple no sólo ha venido reduciendo sus gastos en I+D como porcentaje de sus ventas en los últimos años, sino que además se encuentra muy por debajo de las empresas que muestran los mayores guarismos en dicha variable ${ }^{18}$.

Estas cifras confirman que no es sólo la cantidad, sino la calidad y la orientación de los gastos en I+D lo que determina el desempeño de las compañías líderes de la cadena. Las barreras a la entrada en el eslabón de las empresas con marca están constituidas tanto por los abultados montos destinados a investigación como por su sesgo hacia el diseño de productos estéticos y funcionales, en el marco de estrategias de comercialización y publicidad que den cuenta de tales cualidades. Esto contrasta con los eslabones de fabricación y ensamble, donde los bajos costos salariales, la cercanía a los grandes centros de consumo y la capacidad de responder a diversos segmentos de la industria electrónica constituyen variables críticas para la entrada de nuevas empresas.

\footnotetext{
${ }^{13}$ Según The Wall Street Journal Americas (20/2a), el último lanzamiento de computadoras personales de Apple evidenció que la firma "está convencida de que los mundos de las PC, laptops y aparatos móviles están destinados a converger y que Apple desea ser un catalizador de ese cambio".

14"Los expertos afirman que los vendedores de Apple nunca bombardean a los clientes con cuestiones tecnológicas [..] "Siempre empiezan preguntándole al cliente por su estilo de vida y sus necesidades", afirma Martin Lindstrom, [.] "Lo involucran emocionalmente [..]" (SmartMoney, The Wall Street Journal Americas, 20I2d).

${ }^{15}$ Estos autores atribuyen el éxito del iPod a años de inversión en publicidad cuidadosa y manejo de marca, complementada con la creación de un canal minorista propio (Apple Stores) con buena presencia y visibilidad.

${ }^{16}$ The New York Times (20I2); The Wall Street Journal Americas (20I0).

${ }^{17}$ The Wall Street Journal Americas (2012c).

${ }^{18}$ Así, mientras Microsoft, Nokia, Google y Sony Ericsson tienen una I+D superior al I $2 \%$ de sus ventas, el ratio de Apple resulta inferior al 3\% (información disponible en el sitio de la consultora Asymco).
}

ISSN: 07 I 8-2724. (http://www.jotmi.org)

Journal of Technology Management \& Innovation (c) Universidad Alberto Hurtado, Facultad de Economía y Negocios. 
La cadena electrónica de consumo se encuentra globalmente dispersa y liderada por empresas multinacionales. Dicken (2003) ya sostenía que la producción estaba cada vez más radicada en los países en desarrollo, fundamentalmente en el Este y Sudeste Asiáticos, destacando el crecimiento de empresas surcoreanas (Samsung y Goldstar) y taiwanesas que habían pasado de un esquema de producción a pedido de firmas de EEUU y Japón al desarrollo de marcas y productos propios con instalado prestigio a nivel internacional. La contrapartida de ello fue la salida parcial o total de empresas estadounidenses y europeas de la industria, a través de alianzas estratégicas o cambios de manos con empresas de origen japonés y coreano, fundamentalmente.

Si se analizan las empresas con marca de mayores ventas a nivel mundial, se observa que en 2012 Samsung ha sido la de mayor envergadura en ventas de teléfonos inteligentes (30\% del mercado), seguida por Apple (19,4\%) y Nokia (5\%). En laptops, en cambio, las empresas estadounidenses siguen conservando un liderazgo: en 20I I, H-P y Dell lideraban el mercado global, explicando casi el $30 \%$ de las unidades vendidas. No obstante, se destaca el dinamismo de Apple y la china Lenovo, que hizo que pasaran de sendas cuotas de mercado de $4 \%$ en 2005 a explicar conjuntamente el $20 \%$ de las ventas globales en $20 \mathrm{I}$ l. Finalmente, el mercado de tabletas (de vigoroso crecimiento) en los últimos dos años tiene como líder absoluto a Apple, aunque sus cuotas de mercado se vienen recortando a costa del crecimiento de Samsung y otras firmas que han lanzado sus productos recientemente (Amazon, Nokia).

En tanto, entre las empresas proveedoras de grandes marcas se destaca el liderazgo de Hon Hai, firma taiwanesa que se encarga de elaborar distintos productos (celulares, computadoras, consolas, etc.) y cuya principal localización geográfica se constituye en diferentes regiones de China, aunque también cuenta con plantas en India, el Este Asiático, América Latina (Brasil y México) y Europa Oriental ${ }^{19}$. En línea con lo indicado más arriba, vale la pena destacar también que Hon Hai alterna la mera fabricación con la producción bajo diseños propios, según la empresa cliente y el producto puntual del que se trate.

El ejemplo de Hon Hai es útil para comprender el alcance global de la industria. En ese sentido, cuando Dedrick y Kraemer (2006) sostienen que la fabricación de computadoras "es global, pero el desarrollo y manufactura del producto está concentrado casi por completo en Estados Unidos, Japón, Taiwán y China", debería agregarse que existe también ensamblaje de estos productos en diferentes países del mundo (Brasil, México, países de Europa Centra, etc.). Desde ya, este fenómeno es extrapolable a otros bienes electrónicos, como los teléfonos celulares, que encuentran zona de ensamblado en diferentes regiones del mundo, las que se utilizan fundamentalmente como plataforma de exportación para mercados de consumo regional o para el armado final para el consumo local.

De todos modos y dada la baja generación de valor que tienen las etapas de ensamblado, la perspectiva más trascendente de la industria radica en analizar los movimientos que pueden tener lugar entre los eslabones y procesos principales. En el caso de las laptops, Dedrick y Kraemer indican que en los últimos años Estados Unidos se ha mantenido exclusivamente en la etapa de diseño, Japón ha dejado ciertas etapas de desarrollo para recluirse también en la planeación del producto y Taiwán ha abandonado la fabricación para concentrarse en la fase de desarrollo, siendo China el único país que ha ampliado su participación en la cadena a más de una eslabón, al sumar ciertas actividades de desarrollo a actividades manufactureras que ya venía ejerciendo.

\section{La cadena de la industria farmacéutica}

La industria farmacéutica involucra tanto la producción de medicamentos nuevos como de otros descubiertos años atrás, los que en algunos casos están protegidos por patentes y en otros no. Haakonsson (2009) identifica la existencia de tres sub-cadenas (strands) a escala global: la de productos con marca (comandada por el productor), la de productos genéricos de calidad (comandada por el comprador) y la de productos genéricos de bajo valor (sin comando). Las actividades de investigación e innovación de producto quedan fundamentalmente en mano de los laboratorios líderes de países desarrollados (denominados Big Pharma), verticalmente integrados y elaboradores de productos patentados conocidos como blockbusters ${ }^{20}$. En contraste, las empresas fabricantes de genéricos de calidad no necesariamente llevan a cabo actividades de I+D, y las de genéricos de bajo valor no lo hacen en absoluto.

\footnotetext{
${ }^{19} \mathrm{Se}$ estima que el $40 \%$ de los electrónicos de consumo del mundo son elaborados en fábricas de esta empresa (The New York Times, 2012) y y que su nivel de facturación supera a la efectuada por sus diez principales competidores en forma conjunta (The Wall Street Journal Americas, 20 I0). ${ }^{20}$ Un blockbuster es un medicamento que genera por sí solo mas de 1.000 millones de dólares de ventas anuales. Actualmente, existen en el mundo más de 100 blockbusters.
} 
La taxonomía de Késic (2009) complementa este panorama. El autor distingue tres tipos de compañías farmacéuticas según su "misión baásica, su performance y su desarrollo estratégico": además de los originators (asimilables a los Big Pharma) y los productores de genéricos, reconoce la presencia de los especialistas, que son firmas que realizan investigación básica y desarrollo de productos farmacogenómicos y biotecnológicos. El aporte de Kesic es fundamental tanto por resaltar la existencia de empresas biotecnológicas clasificada en forma separada (lo que revela su creciente importancia a nivel mundial) como por indicar que su clasificación es efectuada por "actividades principales", lo que implica la posibilidad de actividades alternativas al interior de cada tipo de compañía y de estrategias mixtas en el marco de la cadena.

La innovación de producto (elaboración de medicamentos nuevos) en la sub-cadena de productos con marca requiere un buen número de estudios y pruebas (pre-clínicas y clínicas) y aprobaciones de organismos regulatorios previos al lanzamiento al mercado. Terblanche (2008) identifica cinco fases de estudios pre-clínicos (desde la detección de nuevas entidades clínicas hasta el testeo químico, farmacéutico y biológico) y tres fases de estudios clínicos (biodisponibilidad, rangos de dosis y seguridad y eficacia) antes del lanzamiento propiamente dicho. Tanto este autor como Bradfield y ElSayed (2009) coinciden en que las pruebas clínicas son las que involucran los costos más elevados de toda la inversión en I+D del sector ${ }^{21}$. Asimismo, estos procesos suelen ser prolongados, superiores a una década ${ }^{22}$.

En tanto, la I+D realizada por los "especialistas" biotecnológicos exhibe algunos matices con relación a la realizada por los Big Pharma. Primero, los tiempos para que los productos finales alcancen la llegada al mercado son, aunque extensos, menos prolongados que en la producción tradicional ${ }^{23}$.Asimismo, Bradfield y El-Sayed (2009) sostienen que la duración de los procesos de desarrollo de productos en la sub-cadena se está acortando, debido a recientes progresos en los métodos de detección y descubrimiento de drogas. Finalmente, la tasa de éxito ${ }^{24}$ biotecnológica en medicamentos es de $34 \%$, cifra muy superior al $8 \%$ verificado en la farmacéutica tradicional (Bradfield y El-Sayed, 2009).

\footnotetext{
${ }^{21}$ Bradfield y El-Sayed citan un estudio de la Federación Europea de Industrias Farmacéuticas de 2007, que indica que las etapas de testeo clínico constituyen el $43 \%$ del total de fondos volcados a I+D en el sector.Terblanche sostiene que este porcentaje es aún mayor, al oscilar entre el $50 \%$ y el $60 \%$ del costo total de desarrollo de una nueva droga.

${ }^{22}$ Bradfield y El-Sayed sostienen que desde el descubrimiento al patentamiento de un nuevo producto el período promedio se halla alrededor de los 12 años, lo que se ratifica en Kesic (2009), al tiempo que Gutman y Lavarello (201I) exponen datos para Estados Unidos y Europa en los que el lapso abarca entre 10 y 12 años.
}

Junto con la investigación para el descubrimiento de nuevas drogas, la publicidad y marketing es el otro gran destino al que se vuelcan los fondos de los grandes laboratorios. Haakonson (2009) indica que en 2004 las Big Pharma destinaron a actividades de marketing casi el doble de lo que desembolsaron en inversión en I+D, al tiempo que Terblanche (2008) sostiene que las actividades de ventas y marketing constituyen el principal componente de los gastos corporativos de la industria ${ }^{25}$. Desde ya, estos desembolsos se materializan en estrategias diferentes en cada Big Pharma. Esto pone de manifiesto que, aun siendo los medicamentos productos donde el peso de la innovación informacional es imprescindible, la innovación estético-expresiva y la llegada al consumidor resultan insoslayables en la propia dinámica de la competencia.

La actividad farmacéutica detenta la particularidad de ser intensiva en investigación. Datos de la Nacional Science Foundation indican que en $\mathbf{2 0 0 8}$ la industria farmacéutica en EEUU alcanzó un monto de inversión volcada a I+D de unos U\$S 48.000 millones, siendo sólo superada por el segmento de productos electrónicos y computadoras. Los gastos en I+D constituyen, así, una barrera a la entrada insoslayable en el eslabón de concepción y diseño de los productos, encarnado fundamentalmente por las empresas con marca y los especialistas biotecnológicos.

Existen dos elementos interrelacionados que en los últimos años han contribuido a elevar la altura de esta barrera. Se trata de la caída en los niveles de efectividad de la $1+D^{26}$ y los crecientes costos necesarios para desarrollar nuevas drogas $^{27}$. Si a estos elementos se suman los elevados plazos necesarios para la consecución de este proceso (que, como se indicó, suelen superar los 10 años), se termina de configurar un escenario en el que (como indican Terblanche y Bradfield y El-Sayed) no existe relación estadística entre los gastos en I+D y el éxito empresario, ya que la clave no es cuánto se gasta, sino cómo se lo hace.

\footnotetext{
${ }^{23}$ Gutman y Lavarello (20I I) indican que los plazos para alcanzar el lanzamiento al mercado de un producto biosimilar en Europa se extienden entre los 6 y los 9 años.

${ }^{24} \mathrm{Se}$ entiende por tasa de éxito a los productos que pasan las pruebas clínicos y alcanzan el mercado.

${ }^{25} \mathrm{El}$ autor se basa en un informe de PWC que indica que entre 1995 y 2005 los gastos en I+D se elevaron del $15 \%$ al $17,1 \%$ del total, mientras que los volcados a ventas y administración general subieron de $28,7 \%$ a $33,1 \%$.

${ }^{26}$ Bradfield y El-Sayed (2009) sostienen que el número de nuevas entidades químicas cayó desde 100 anuales en la década de 1960 hasta las 20/30 registradas en los primeros años del presente siglo.

${ }^{27}$ Bradfield y El-Sayed indican que en EE.UU. los costos desde el descubrimiento hasta la aprobación de una nueva droga por parte de la FDA se elevaron desde U\$S I54 millones en 1976 a U\$S 800 millones en 2000 , y que incluso ya se ha entrado plenamente en la era de las "drogas de los mil millones".
} 
Dado que los gastos en publicidad y comercialización superan a los destinados a I+D, resulta evidente que aquellos también constituyen una barrera a la entrada muy significativa. Más aún, es allí donde reside la principal fortaleza de las Big Pharma, toda vez que la construcción de marcas y su trayectoria en el mercado construyen la inercia que suele operar para la aceptación de productos sustitutos. Esta es también la principal debilidad de las firmas biotecnológicas, que además deben contar con flexibilidad para adaptarse a contextos regulatorios con normas de aprobación disímiles (Gutman y Lavarello, 20I I). Pero como los plazos, costos y tasa de fracaso para desarrollo de biosimilares son inferiores a los experimentados por los laboratorios tradicionales, emerge una necesidad recíproca entre las empresas con marca y los especialistas que conduce a que formen alianzas (cooperando en ciertos proyectos) y compitan en determinados productos al mismo tiempo (Kesic, 2009).

Por otra parte, Haakonsson (2009) sostiene que en el caso de los "genéricos de calidad" las principales barreras están constituidas por el precio, la reputación, la calidad del bien y las aprobaciones internacionales de los productos. En tanto, en los "genéricos de bajo valor" son el precio y el cumplimiento de los estándares mínimos dispuestos por la OMS los elementos que más condicionan la posibilidad de competir en la sub-cadena, al tiempo que la eficacia del producto pasa a un segundo plano. Kesic (2009) coincide en que la competencia entre fabricantes de genéricos radica en los precios de sus productos, añadiendo que el desenvolvimiento de un mercado mundial de genéricos depende de en última instancia de la legislación vigente (regulación internacional y de los sistemas de propiedad intelectual de cada país).

Con todo, los genéricos son naturalmente los productos que implican menores costos de producción. Bradfield y El-Sayed (2009) sostienen que en EEUU el costo estimado de desarrollar una droga genérica es de U\$S I millón, lo que les permite ser vendidas hasta un $80 \%$ por debajo del precio de las drogas equivalentes comercializadas por las Big Pharma. Dicho monto también es sensiblemente inferior al involucrado en el desarrollo de biosimilares, que en el mercado europeo oscila entre los U\$S 75 y 150 millones por medicamento (Gutman y Lavarello, 20 I I). Esta comparación brinda una clara perspectiva del rol disímil que juega la innovación de producto en cada caso.
Los principales laboratorios tienen su sede central en los países desarrollados. De las 20 firmas que lideraron las ventas globales de medicamentos en 2011, 8 son estadounidenses (incluyendo a Pfizer y Merck, primera y tercera respectivamente), 8 de Europa Occidental, 3 japonesas (ninguna de las cuales está entre las 10 primeras) y una israeli ${ }^{28}$. Las diez empresas con mayor facturación explicaron en forma conjunta el $43,4 \%$ de las ventas globales de la industria, mientras que las veinte primeras alcanzaron a superar el $60 \%$ de la facturación global. La concentración geográfica es aún mayor si se observan los datos de las exportaciones globales ${ }^{29}$.

Este liderazgo coexiste con la presencia de empresas de cierta envergadura en países emergentes o donde la industria farmacéutica tuvo un desarrollo tardío. Haakonsson (2009) destaca que los productores de genéricos de calidad están globalmente dispersos y que India, China, Singapur y Brasil son casos particulares de países donde ciertas empresas nacionales se han desenvuelto hasta convertirse en "proveedores de paquete completo" de multinacionales que terciarizaron parte de sus procesos. Asimismo, resalta el hecho de que en los últimos años la capacidad exportadora de India se ha incrementado hacia los países desarrollados y también hacia África. Por su parte, Gutman y Lavarello (20II) destacan el desarrollo de capacidades productivas en el segmento de biosimilares en China, India y Corea del Sur. Uno de las tendencias recientes en la cadena radica en el ingreso de empresas multinacionales en la sub-cadena de genéricos bajo distintas estrategias (absorciones, acuerdos de producción o comercialización, etc.), concentrándose en particular en los mercados emergentes. Análogamente, firmas de países emergentes encaran estrategias novedosas, como la penetración en mercados de países desarrollados, la adquisición de (o acuerdos con) firmas de dichos países y hasta la incorporación de actividades de I+D; el caso más destacado, en ese sentido, es el de las empresas indias. Estas iniciativas determinan el panorama de una industria en pleno cambio, en la que las estrategias empresariales tienden a ser cada vez más diversificadas.

\footnotetext{
${ }^{28}$ Información disponible en el sitio web de la consultura IMS Health (www. imshealth.com).

${ }^{29} \mathrm{En} 2008$, los países desarrollados fueron responsables del $90,5 \%$ de las ventas externas de medicamentos, siendo el $80 \%$ de las mismas explicado por Europa (UE-15 más Suiza) y el $8 \%$ por Estados Unidos (Zhang et.al., 2012).
}

ISSN: 07 I8-2724. (http://www.jotmi.org) 
La reorientación llevada a cabo por las Big Pharma tiene un alcance incluso superior al expuesto: los vencimientos en las patentes de los blockbusters y la falta de bienes sustitutos (factores a los que se suman los crecientes costos de investigación y su mayor tasa de fracaso) motivaron un viraje desde medicamentos para mercados amplios (colesterol, diabetes, etc.) a segmentos específicos como medicamentos huérfanos, OTC y vacunas (Tovar, 2009). En sintonía con lo arriba expuesto, el panorama del viraje de las empresas líderes se completa con su acercamiento hacia la actividad biotecnológica, lo que se materializó en el lanzamiento de nuevas unidades productivas, adquisición de empresas ya instaladas o acuerdos de financiamiento e investigación específicos (Bradfield y El-Sayed, 2009; Gutman y Lavarello, 20II).

En cualquier caso, debe señalarse que la diversificación de estrategias por parte de los distintos agentes de la cadena puede encontrar ciertas limitaciones en algunos puntos. La renuencia de las grandes empresas farmacéuticas a investigar sobre enfermedades raras pero serias y las capacidades mínimas requeridas por los países desarrollados para abocarse a una industria de semejante complejidad (Gutman y Lavarello, 20II) son elementos que relativizan el alcance de las reorientaciones expuestas. En sintonía con ello, distintas voces han planteado dudas acerca de las verdaderas capacidades con que cuentan los sectores farmacéuticos de algunos países en desarrollo. El caso de China ha sido bastante citado en ese sentido, dado sus bajos niveles relativos de I+D en esta industria ${ }^{30}$ (Zhang et al, 2012).

\footnotetext{
${ }^{30} \mathrm{La}$ intensidad de I+D del sector fue en 2006 superior al $40 \%$ en EE.UU. e Inglaterra, al $30 \%$ en Japón y Francia y al $20 \%$ en Alemania, mientras que en China no alcanzó el 5\% en 2007.
}

\section{Conclusiones}

A modo de reflexión final, la evidencia que surge de los tres casos de estudio tiende a confirmar (al menos de manera parcial, dado el carácter no exhaustivo del estudio) nuestra hipótesis inicial: la existencia de un tendencia propia del nuevo capitalismo a segmentar la actividad económica mundial al interior de cadenas globales de valor entre, por un lado, un conjunto de segmentos que están en la vanguardia de la creación de nuevo conocimiento y otros intensivos en el uso y adaptación de conocimiento existente. No obstante, las formas en que se despliega esta tendencia son altamente heterogéneas, según la naturaleza de las barreras a la entrada en las actividades de innovación y de producción.

En la cadena de indumentaria, el proceso de innovación tiene un bajo componente informacional y un alto contenido estético-expresivo (construcción de marcas, instauración de modas), al tiempo que la producción refleja un escaso grado de automatización (sobre todo en confección de prendas) y requerimientos laborales simples desde el punto de vista cognitivo. En la cadena farmacéutica, la innovación de producto tiene un sesgo informacional de alta complejidad acompañada de una creciente dimensión estético-expresiva (a partir de la fuerte publicidad requerida por vencimiento de patentes y competencia de genéricos), mientras que la fabricación sólo es demandante de innovaciones de tipo adaptativas. Por último, en electrónica de consumo la innovación también está fuertemente asentada en un componente informacional (rejuvenecimiento de productos y convergencia de funciones), aunque también dotada de un componente estético-expresivo que se manifiesta en la necesidad de productos funcionales y sofisticados; la producción, por su parte, detenta requerimientos cognitivos menores, aunque mayores en la fabricación de componentes críticos y desarrollo de productos que en la etapa de ensamblaje.

Con todo, la integración global de la producción supone una redefinición en la naturaleza de las asimetrías en la economía mundial. A pesar de la existencia de elementos de contra-tendencia (cierta difusión de actividades de I+D hacia la periferia y mayores requerimientos en estándares técnicos para las actividades de fabricación y ensamblaje), lo central radica en el proceso histórico de elevación de las barreras a la entrada en las actividades de innovación y de caída en las de producción. Este fenómeno se verifica simultáneamente con una diferenciación estructural de los sistemas de innovación sobre los que se construyen las capacidades competitivas de cada territorio. Por consiguiente, el concepto de cadena global de valor debe entenderse no sólo como herramienta conceptual para el diseño de políticas de desarrollo productivo, sino también a partir de su rol en el establecimiento y reproducción de jerarquías entre territorios que evidencian capacidades de innovación asimétricas. 


\section{Referencias}

ABERNATHY, W. y UTTERBACK, J. (1978). Patterns of Innovation in Technology. Technology Review, v 7, n. 80, p. 40-47.

ALTENBURG, T., SCHMITZ, H. y STAMM, A. (2008). Breakthrough? China's and India's Transition from Production to Innovation. World Development, v. 36, n. 2, p. 325-344.

BRADFIELD, R.y EL-SAYED, H. (2009). Four scenarios for the future of the pharmaceutical industry. Technology Analysis \& Strategic Management, v. 2I, n. 2, p. 195-212.

BELL, M. y ALBU, M. (1999). Knowledge Systems and Technological Dynamism in Industrial Clusters in Developing Countries.World Development, v. 27, n. 9, p. 17I5-1734.

DEDRICK, J.y KRAEMER, K. (2009). Redes de conocimiento global en el desarrollo de nuevos productos: la industria de las PC portátiles, en DABAT, A. y RODRIGUEZ VARGAS, J., coord. Globalización, conocimiento y desarrollo. La nueva economía global del conocimiento, estructura y problemas. México D.F. Universidad Nacional Autónoma de México Miguel Ángel Porrúa.

DEDRICK, J., KRAEMER, K. y LINDEN, G. (2009). Who profits from innovation in global value chain?: a study of the iPod and notebook PCs. Industrial and Corporate Change, Vol. 19, No. I, pp. 8I-I I6.

DICKEN, P. (2003). Global Shift. Transforming the global economy. New York, The Guilford Press.

DIEAUIDE, B., PAULRE, B. y VERCELLONE, C. (2007). Introducción al capitalismo cognoscitivo, en RIVERA RIOS, M. y DABAT,A., coord. Cambio histórico mundial, conocimiento y desarrollo. México D.F., Universidad Nacional Autónoma de México (UNAM) - Casa Juan Pablos Centro Cultural.

GEREFFI, G. (1999). International trade and industrial upgrading in the apparel commodity chain. Journal of International Economics, n. 48 , p. 37-70.

GEREFFI, G. (200I). Las cadenas productivas como marco analítico para la globalización. Problemas del Desarrollo. México D.F, v. 32, n. 125.

GEREFFI, G. y MEMEDOVIC, O. (2003). The Global Apparel Value Chain. What Prospects for Upgrading by Developing Countries?. Vienna, United Nations Industrial Development Organization (UNIDO).
GIBBON, P. (2008). Governance, Entry Barriers, Upgrading: A Re-Interpretation of Some GVC Concepts from the Experience of African Clothing Exports. Competition \& Change, v. 12, n. I, p. 29-48.

GUTMAN, G. y LAVARELLO, P. (20II). Formas de organización de las empresas biotecnológicas en el sector farmacéutico argentino. Desarrollo Económico, v. 5I, n. 20 I.

HAAKONSSON, S. (2009). The Changing Governance Structures of the Global Pharmaceutical Value Chain. Competition \& Change, v. I3, n. I, p. 75-95.

KAPLINSKY, R. (2000). Globalization and Unequalization: What can Be Learned from Value Chain Analysis. The Journal of Development Studies, v. 37, n. 2.

KAPLINSKY, R. (2005). Globalization, poverty and inequality. Between a rock and a hard place. Cambridge, Polity Press.

KESIC, D. (2009). Strategic analysis of the world pharmaceutical industry. Management, v. I4, n. I, p. 59-76.

LASH, S. y URRY, J. (1998). Economías de signo y espacio. Buenos Aires, Amorrortu.

PADILLA, M., CORDERO, M., HERNANDEZ, R.,y ROMERO, I. (2008). Evolución reciente y retos de la industria manufacturera de exportación en Centroamérica, México y República Dominicana: una perspectiva regional y sectorial. México, CEPAL, Serie Estudios y Perspectivas, n. 95.

RIVERA, M.A. (2005). Capitalismo informático, cambio tecnológico y desarrollo nacional. México: Universidad de Guadalajara, Universidad Nacional Autónoma de México, UCLA Program on Mexico, PRFOMEX - Juan Pablos Editor.

STANLEY, L. (20II). La inserción de América del Sur en las cadenas globales de valor, en PROCHNIK, V., coord. La inserción de América Latina en las cadenas globales de valor. Red Mercosur, n. 19., p. 69-196.

STURGEON, T. (2002). Modular Production Networks: A New American Model of Industrial Organization. Cambridge, USA, Industrial Performance Center. Massachusetts Institute of Technology (MIT).

SZTULWARK, S., MIGUEZ, P. y JUNCAL, S. (201I). Conocimiento y valorización en el capitalismo industrial. Revista de Historia de la industria, los servicios y las empresas en América Latina, Buenos Aires, Argentina, n. 9. 
TERBLANCHE, N. (2008). New pharmaceutical product development: Barriers to overcome and opportunities to exploit. Journal of Commercial Biotechnology. v. 14, n. I3, p. 20I-2I 2 .

THE ECONOMIST (20I I). Slicing an Apple. I0/8/20I I.

THE NEW YORK TIMES (20I2). How the U.S. Lost Out on iPhone Work. 2I/I/20I 2 .

THEWALL STREET JOURNALAMERICAS (20I I). En busca del nuevo Lipitor, Pfizer se centra en las drogas de nicho. $30 / 8 / 2011$.

THE WALL STREET JOURNAL AMERICAS (2012a). Apple quiere que sus Macs se parezcan más a sus iPhones. 17/2/2012.

THE WALL STREET JOURNAL AMERICAS (2012b). EI error más caro en la historia de Nokia. 20/7/2012.

THE WALL STREET JOURNAL AMERICAS (20I2c). Cómo Japón perdió su dominio en los electrónicos de consumo. 16/8/2012.

THE WALL STREET JOURNAL AMERICAS (20I2d). Diez cosas que Apple nunca le dirá. 30/8/2012.

THE WALL STREET JOURNAL AMERICAS (20I2e). H-P muestra su nueva cara. 18/9/20I2.

TOVAR, M. (2009). Matices: Las grandes farmacéuticas piensan ahora en pequeño. En: http://www.elglobal.net/ elglobal/articulo.aspx?idart=454579\&idcat=633\&tipo $=2$.

ZHANG, Y., LI, D., YANG, C., y DU, Q. (20I2). On the Value Chain and International Specialization of China's Pharmaceutical Industry. Washington D.C., United States International Trade Commission (USITC). 